\title{
L'addition de lactose et de vitamine C dans les laits de croissance diminue la qualité nutritionnelle des protéines
}

\author{
Inès Birlouez-Aragon ${ }^{\mathrm{a} *}$, Pascal Sabat ${ }^{\mathrm{a}}$, Bérengère Lutz ${ }^{\mathrm{a}}$, \\ Juliette Leclère ${ }^{\mathrm{a}}$, Marina Nicolas ${ }^{\mathrm{b}}$ \\ a Laboratoire de chimie analytique, Institut national agronomique, \\ 16, rue Claude-Bernard, 75231 Paris cedex 05, France \\ ${ }^{\mathrm{b}}$ Cneva, 10, rue Pierre-et-Marie-Curie, 94704 Maisons-Alfort, France
}

(Reçu le 12 mai 1999 ; accepté le 23 août 1999)

\begin{abstract}
Addition of lactose and vitamin $\mathrm{C}$ to growth milk reduces the nutritional quality of proteins. Addition of lactose to growth milk $\left(+20-30 \mathrm{~g} \cdot \mathrm{L}^{-1}\right)$ is responsible for an increase in the Maillard reaction which induces a loss in nutritional value of protein and the development of brown compounds. This reaction is further reinforced by the iron-vitamin $\mathrm{C}$ mixture, because of the production of free radical species during heat treatment. However, iron supplementation is one of the nutritional interest of growth milk, whereas lactose addition does not seem to trigger any nutritional benefit for children. Furthermore, despite the higher iron biodisponibility allowed by simultaneous vitamin $\mathrm{C}$ addition, it seems better not to add the vitamin $\mathrm{C}$ to avoid its interaction with iron. A new UHT growth milk formula, without addition of lactose and vitamin $\mathrm{C}$, has been compared to a formula containing both nutrients and produced using the same UHT heat treatment. We provide evidence of a very significant improvement in the protein quality of the new formula as compared with the classic growth milk formula, regarding both the early ( $25 \%$ lower) and advanced Maillard reaction ( $400 \%$ less), measured as furosine and fluorescence at 330 and $420 \mathrm{~nm}$ respectively. Furthermore, whereas the tryptophan and tyrosine contents of the soluble proteins are $30 \%$ lower in the classic formula than in cow's milk, they are unchanged in the new formula. In conclusion, we demonstrate that omission of lactose and vitamin $\mathrm{C}$ enrichment in growth milk allows a protein quality similar to that usually found in cow's milk to be restored, despite the presence of iron. (C) Inra/Elsevier, Paris.
\end{abstract}

growth milk / lysine bioavailability / tryptophan / Maillard reaction / iron-ascorbate

Résumé - L'addition de lactose aux laits de croissance $\left(+20-30 \mathrm{~g} \cdot \mathrm{L}^{-1}\right)$ entraîne une augmentation de la réaction de Maillard qui a pour effet de diminuer la valeur nutritionnelle des protéines et de favoriser le développement d'une coloration brunâtre. Cette réaction est également activée par le mélange fer-ascorbate du fait de la production d'espèces réactives de l'oxygène au cours du traitement thermique. L'enrichissement en fer constitue l'un des intérêts nutritionnels des laits de croissance, alors

* Correspondance et tirés à part. BirlouezIE@aol.com 
que l'addition de lactose ne semble pas justifiée sur le plan nutritionnel. De plus, il nous semble préférable de ne pas ajouter de vitamine $C$ pour éviter son interaction avec le fer. Nous comparons une nouvelle formule de lait de croissance sans addition de lactose et vitamine $\mathrm{C}$ à un lait de croissance de composition classique et soumis au même traitement de stérilisation UHT. Nous montrons une très nette amélioration de la qualité nutritionnelle des protéines dans la nouvelle formule par rapport à son homologue non modifié, à savoir une diminution de $25 \%$ de la réaction précoce de Maillard (furosine) et de $400 \%$ de la réaction avancée (fluorescence à $340 / 430 \mathrm{~nm}$ ). De plus, les teneurs de tryptophane et tyrosine des protéines solubles sont similaires à celles attendues dans un lait de vache, alors que celles-ci sont abaissées de $30 \%$ dans le lait classique de croissance. En conclusion, l'abandon de l'enrichissement en lactose et en vitamine $C$ constitue une voie d'amélioration de la qualité des laits de croissance. OInra/Elsevier, Paris.

\section{lait de croissance / lysine biodisponible / tryptophane / réaction de Maillard / fer-ascorbate}

\section{INTRODUCTION}

Le développement des laits de croissance enrichis en fer a été motivé par le constat d'une forte prévalence de l'anémie chez le jeune enfant [11]. Du fait de la faible biodisponibilité du fer dans le lait de vache (4 à $6 \%$ ) [8], par rapport au lait de femme, une quantité 10 fois supérieure est ajoutée dans le lait de croissance. Ceci assure aux enfants de $1-3$ ans consommant $500 \mathrm{~mL}$ de lait de croissance par jour un apport satisfaisant, que le reste de l'alimentation ne permet pas toujours. Une baisse de plus de trois fois de la prévalence des anémies, de 30 à 2-11\%, a en effet été mesurée chez les enfants de 15 mois recevant une formule lactée enrichie en fer par rapport à ceux alimentés avec du lait de vache [9]. Les laits de croissance permettent ainsi d'assurer une transition entre lait de suite et lait de vache, alors que parallèlement l'alimentation se diversifie. La composition des laits de croissance demeure relativement proche de celle des laits de suite (teneurs en fer, vitamine C, acides gras essentiels, lactose...) ; la concentration en protéines est toutefois plus élevée (28-32 g. $\mathrm{L}^{-1}$ au lieu de $\left.20-22 \mathrm{~g} \cdot \mathrm{L}^{-1}\right)$; elle est similaire à celle du lait de vache.

Diverses études $[2,3,12,16]$ ont démontré la difficulté de maîtriser, durant la stérilisation et la conservation, les réactions d'oxydation radicalaire dues au mélange fer-vitamine $\mathrm{C}$ ainsi que la réaction de Maillard due au lactose. Un travail antérieur [3] avait mis en évidence un taux de blocage trois fois plus important de la lysine des protéines totales et une réduction de $30 \%$ du Trp peptidique dans la fraction des laits de croissance soluble à $\mathrm{pH} 4,6$. Des observations tout à fait similaires ont ensuite été rapportées pour les laits infantiles [5]. La perte de lysine est partiellement compensée à la fois par la richesse des protéines du lait de vache en cet acide aminé essentiel et par la teneur importante de protéines ingérées par rapport aux besoins. Cependant, les conséquences de la réaction, telles que l'apparition de composés avancés de Maillard mal connus et la libération de radicaux libres [2] pouvant attaquer les acides aminés sensibles à l'oxydation (tryptophane, tyrosine, histidine et acides aminés soufrés) [22] peuvent laisser suspecter une altération globale de la qualité des protéines du lait. Des études in vitro et chez l'animal ont en effet montré une perte nette de la digestibilité et de la biodisponibilité de la lysine et des acides aminés soufrés dans les protéines de lait infantile soumises à un traitement UHT ou de stérilisation [18, 19]. De plus, les nourrissons recevant des formules infantiles présentent des teneurs de tryptophane circulant toujours inférieures à celles des nourrissons allaités [7]. Ceci est lié à la présence d'une concentration double 
d' $\alpha$-lactalbumine, très riche en Trp, dans le lait de femme par rapport au lait de vache. Cependant, la concentration près de deux fois plus importante de protéines totales des formules (autour de $20 \mathrm{~g} \cdot \mathrm{L}^{-1}$ ) par rapport au lait de femme $\left(10 \mathrm{~g} \cdot \mathrm{L}^{-1}\right)$ compense la déficience en cet acide aminé. On doit donc en conclure que la biodisponibilité du Trp est diminuée dans les formules infantiles.

Une adaptation des formules de laits de croissance est donc nécessaire pour améliorer la qualité des protéines. Nous évaluons ici l'influence de l'enrichissement en lactose et en vitamine $\mathrm{C}$ sur la qualité des protéines avant d'envisager son abandon. On assiste déjà de manière de plus en plus généralisée à un remplacement dans les formules infantiles du lactose par des maltodextrines à moindre pouvoir réducteur [6] L'absence de vitamine $C$ devrait de plus éviter la libération de radicaux libres catalysée par le fer.

Nous comparons donc la qualité des protéines de trois laits traités sur le même système de stérilisation UHT indirect et conservés trois mois dans le même type d'emballage : 1) le lait de croissance modifié (LCM) selon ces deux critères (lactose et vitamine C), 2) un lait de croissance classique (LC), de composition similaire à celle de la moyenne des laits de croissance présents sur le marché, et 3) un lait de vache non supplémenté (LV). Divers marqueurs de la réaction de Maillard ont été analysés au cours de trois mois de conservation. À trois mois (moitié de la durée de vie pour les laits de croissance), les teneurs en tryptophane et tyrosine des protéines totales et solubles à $\mathrm{pH} 4,6$ ont été déterminées, ainsi que la composition protéique de la fraction soluble à $\mathrm{pH} 4,6$. Les résultats de ces analyses sont ensuite comparés à ceux obtenus dans les laits de croissance du marché.

\section{MATÉRIEL ET MÉTHODES}

Nactalia (Nantes, France) nous a fourni chaque mois, durant trois mois, trois packs de lait conservés d'une part à $4{ }^{\circ} \mathrm{C}$, d'autre part à $25^{\circ} \mathrm{C}$ des trois laits suivants :

- 1 lait UHT $1 / 2$ écrémé de grande consommation (LV, témoin),

- 1 lait croissance (LC),

- le lait croissance modifié sans ajout de lactose et de vitamine C (LCM) qui est obtenu par ailleurs selon une production biologique.

Les compositions théoriques des laits sont présentées dans le tableau $I$ en distinguant d'une part la composition en macronutriments qui est donnée pour les trois laits et d'autre part la quantité des divers micronutriments rajoutés dans les deux laits de croissance, telle que mentionnée sur l'étiquetage.

Les packs de lait étaient ouverts le jour même et les différentes méthodes ont été appliquées sur l'un ou les trois exemplaires d'un même échantillon.

À titre de comparaison, les diverses marques de laits de croissance du commerce ont été analysées.

\subsection{Dosage de la furosine}

La furosine est produite par l'hydrolyse acide de la $\mathrm{N}_{\varepsilon}$-lactulosyl-lysine. Un aliquot de lait contenant $50 \mathrm{mg}$ de protéines est hydrolysé en présence d'HCl $7,8 \mathrm{~N}$ à $110^{\circ} \mathrm{C}$ pendant $23 \mathrm{~h}$. La furosine libérée est séparée par HPLC selon la méthode de Resmini et al. [17] sur une colonne en phase inverse à paire d'ions (colonne spécifique furosine, Altech, Templemars, France) et mesurée en UV $(280 \mathrm{~nm})$ après filtration de l'hydrolysat, dilution et extraction sur filtre. L'analyse a été réalisée en double sur un échantillon de chaque type de lait. Le coefficient de variation de répétabilité est de $4 \%$.

\subsection{Mesure des produits avancés de Maillard}

Les produits avancés de Maillard sont mesurés grâce à leur fluorescence spécifique (excitation $350 \mathrm{~nm}$, émission $440 \mathrm{~nm}$ ) selon la méthode FAST [4]. Les caséines et protéines du lactoserum dénaturées sont précipitées à $\mathrm{pH}$ 4,6 par addition de neuf volumes de tampon acétate de sodium $0,1 \mathrm{~mol} \cdot \mathrm{L}^{-1}$ à $\mathrm{pH} 4,6$. Le surnageant de centrifugation est transvasé dans une cuve quatre faces optiques jetable en acryle (Sarstedt, Ivrysur-Seine, France) et la fluorescence du trypto- 
Tableau I. Composition des laits d'après l'étiquetage.

Table I. Milk composition according to labelling.

\begin{tabular}{|c|c|c|c|}
\hline & $\begin{array}{c}\text { LC } \\
\text { Pour } 100 \mathrm{~mL}\end{array}$ & $\begin{array}{c}\text { LCM } \\
\text { Pour } 100 \mathrm{~mL}\end{array}$ & $\begin{array}{c}\text { LV* } \\
\text { Pour } 100 \mathrm{~mL}\end{array}$ \\
\hline Protéines (g) & 3 & 3,1 & 3,2 \\
\hline $\begin{array}{l}\text { Glucides }(\mathrm{g}) \\
\text { dont lactose }(\mathrm{g}) \\
\text { maltodextrines }(\mathrm{g})\end{array}$ & $\begin{array}{l}7,2 \\
6,2 \\
1\end{array}$ & $\begin{array}{l}4,5 \\
4,5 \\
0\end{array}$ & $\begin{array}{l}4,6 \\
4,6 \\
0\end{array}$ \\
\hline $\begin{array}{l}\text { Lipides (g) } \\
\text { dont ac. Linoléique (mg) } \\
\text { ac. Linolénique (mg) } \\
\% \text { MG laitière } \\
\text { Calcium (mg) } \\
\text { Phosphore (mg) }\end{array}$ & $\begin{array}{l}2,55 \\
195 \\
30 \\
80 \\
119 \\
80\end{array}$ & $\begin{array}{l}2,70 \\
250 \\
30 \\
80 \\
119 \\
80\end{array}$ & $\begin{array}{l}1,6 \\
\text { nd } \\
\text { nd } \\
100 \\
114 \\
85\end{array}$ \\
\hline $\begin{array}{l}\text { Nutriments rajoutés au lait } \\
\text { Zine (mg) } \\
\text { Fer (mg) }\end{array}$ & $\begin{array}{l}0 \\
1\end{array}$ & $\begin{array}{l}1,5 \\
1,5\end{array}$ & \\
\hline $\begin{array}{l}\text { Vit A }(\mu \mathrm{g}) \\
\text { VitD3 }(\mu \mathrm{g}) \\
\text { VitE }(\mathrm{mg}) \\
\text { Vitamin C }(\mathrm{mg}) \\
\text { Vit B1 }(\mathrm{mg}) \\
\text { Vit B2 }(\mathrm{mg}) \\
\text { Vit B5 }(\mathrm{mg}) \\
\text { Vit B6 }(\mathrm{mg}) \\
\text { Vit B9 }(\mathrm{mg}) \\
\text { Vit B12 }(\mathrm{mg}) \\
\text { Vit PP }(\mathrm{mg})\end{array}$ & $\begin{array}{l}29 \\
1,2 \\
0,65 \\
1,8 \\
0,04 \\
0,12 \\
0,2 \\
0,04 \\
5,5 \\
0,3 \\
0,1\end{array}$ & $\begin{array}{l}29 \\
1,2 \\
0,65 \\
0 \\
0,04 \\
0,12 \\
0,3 \\
0,04 \\
5,5 \\
0,3 \\
0,1\end{array}$ & \\
\hline
\end{tabular}

* source base de données Régal (Origine : Table Regal).

phane (290/340 nm ; excitation/émission) et des produits de Maillard (350/440 nm) est mesurée sur un spectrofluorimètre Spex (Jobin-Yvon, Longjumeau, France). La mesure FAST correspond au rapport entre les fluorescences des produits de Maillard et du tryptophane. L'analyse a été réalisée sur les trois packs de chaque type de lait. Le coefficient de variation de répétabilité de la méthode est de 2,5\%.

L'intensité relative de fluorescence du Trp (FW) est mesurée par le rapport entre la fluorescence et la concentration de protéines dans le surnageant (PS en $\left.\mathrm{g} \cdot \mathrm{L}^{-1}\right)$, elle-même quantifiée par la méthode de Lowry. Pour éviter l'effet des fluctuations de la lampe sur l'intensité de fluorescence, l'intensité relative est donnée en pourcentage, en se basant sur la pente de la droite de régression linéaire obtenue pour les laits UHT entre la fluorescence du Trp et la concentration en protéines solubles [4] :

$\mathrm{FW}=\mathrm{a}(\mathrm{PS}-\mathrm{b})$ où $\mathrm{a}$ est la pente de la droite ramenée à 100 arbitrairement. La constante b, égale à $0,92 \mathrm{~g} \cdot \mathrm{L}^{-1}$ en moyenne, représente la concentration en protéose-peptone qui ne contient pas de Trp.

\subsection{Dosage du tryptophane et de la tyrosine}

Ces deux acides aminés aromatiques ont été dosés dans les protéines totales et dans les surnageants acétate contenant les protéines peu dénaturées et solubles à $\mathrm{pH}$ 4,6 d'après la méthode de Delhaye et Landry [15]. Suivant les cas, $330 \mu \mathrm{L}$ de lait ou $1,6 \mathrm{~mL}$ de surnageant acétate 
sont hydrolysés en milieu basique par addition de $840 \mathrm{mg}$ d'hydroxyde de barium. L'hydrolyse se fait à $110^{\circ} \mathrm{C}$ pendant $16 \mathrm{~h}$ en milieu humide (autothermos). Après refroidissement, la solution est acidifiée par addition d' $\mathrm{HCl}$ jusqu'à obtention d'un $\mathrm{pH}$ de 3 environ. L'hydrolysat légèrement acide est alors transvasé dans une fiole de $25 \mathrm{~mL}$ et dilué avec de l'eau Millipore. Après filtration, le filtrat est injecté sur une colonne Spherisorb C18. L'éluant est un mélange $75 / 25(\%)$ d'acétate d'ammonium $14,5 \mathrm{mmol} \cdot \mathrm{L}^{-1}$ et de méthanol, ajusté après mélange à $\mathrm{pH} 4,5$ avec de l'acide formique. Le débit est de $1 \mathrm{~mL} \cdot \mathrm{min}^{-1}$. La détection est réalisée par fluorescence aux longueurs d'onde maximales pour ces deux acides aminés (270/310 et $280 / 350 \mathrm{~nm}$ respectivement). Ces analyses ont été réalisées exclusivement sur les échantillons de lait conservés trois mois, en double exemplaire.

Par ailleurs, la teneur en protéines solubles a été évaluée par la méthode de Lowry et la composition en $\beta$-lactoglobuline et en $\alpha$-lactalbumine quantifiée par HPLC au moyen d'une colonne d'exclusion. La colonne de verre, de type Waters Protein Pak 200 W $(8 \times 300 \mathrm{~mm})$ permet la séparation de protéines entre 500 et $60000 \mathrm{~g} \cdot \mathrm{mol}^{-1}$. L'éluant est constitué d'un tampon acétate d'ammonium $80 \mathrm{mmol} \cdot \mathrm{L}^{-1}, \mathrm{pH}$ 6,2 à $0,8 \mathrm{~mL} \cdot \mathrm{min}^{-1}$. Les pics sont détectés à la fois par fluorescence à $280 / 337 \mathrm{~nm}$ (longueurs d'onde de fluorescence du Trp) et par absorbance à $280 \mathrm{~nm}$. L'étalonnage est réalisé à partir de solutions d' $\alpha$-lactalbumine et de $\beta$-lactoglobuline (Sigma, Saint-Quentin-Fallavier, France) préparées dans le tampon acétate de sodium à
$0,1 \mathrm{~mol} \cdot \mathrm{L}^{-1}$ et $\mathrm{pH} 4,6$, à une concentration de $0,25 \mathrm{~g} \cdot \mathrm{L}^{-1}$. Le coefficient de variation de répétabilité de la mesure est de $4 \%$.

\subsection{Dosage de la vitamine $C$}

Un aliquot de lait est immédiatement mis en présence d'un volume d'acide métaphosphorique et centrifugé pour séparer le surnageant contenant la vitamine $\mathrm{C}$. La somme « acide ascorbique et acide déhydroascorbique » est dosée après oxydation de l'acide ascorbique en acide déhydroascorbique par l'iode. Ce produit est alors dérivé par la dimethylphenylènediamine et détecté en fluorescence $(360 / 440 \mathrm{~nm})$ après séparation HPLC selon la méthode de Tessier et al. [20]. Le dosage est réalisé le jour même. Le coefficient de répétabilité de la méthode est de 3,5\%. Les trois packs de chaque type de lait ont été analysés.

\section{RÉSULTATS}

\section{1. Évolution de la vitamine $\mathbf{C}$ en fonction de la température et du temps de conservation (tableau II)}

La concentration de vitamine $C$ est légèrement supérieure à trois mois dans LCM que dans LV, malgré l'absence d'enrichis-

Tableau II. Teneur en vitamine $\mathrm{C}\left(\mathrm{mg} \cdot \mathrm{L}^{-1}\right)$ des différents laits au cours de leur conservation.

Table II. Vitamin C concentration $\left(\mathrm{mg} \cdot \mathrm{L}^{-1}\right)$ of the milk samples as a function of preserving.

\begin{tabular}{lccrr}
\hline $\begin{array}{l}\text { Conservation } \\
\text { (mois) }\end{array}$ & $\begin{array}{c}\text { Température } \\
\left({ }^{\circ} \mathrm{C}\right)\end{array}$ & $\begin{array}{c}\mathrm{LV} \\
\mathrm{N}=3\end{array}$ & $\begin{array}{c}\mathrm{LC} \\
\mathrm{N}=3\end{array}$ & $\begin{array}{c}\mathrm{LCM} \\
\mathrm{N}=2^{*}\end{array}$ \\
\hline 1 & 4 & $9,4 \pm 1,3$ & $17,6 \pm 3,1$ & $12,8 \pm 2,8$ \\
1 & 25 & $8,8 \pm 1,5$ & $9,4 \pm 0,3$ & $7,3 \pm 0,3$ \\
2 & 4 & $3,2 \pm 0,4$ & $6,2 \pm 0,7$ & $4,0 \pm 1,7$ \\
2 & 25 & $1,1 \pm 0,2$ & $0,7 \pm 0,6$ & $0,9 \pm 0,5$ \\
3 & 4 & $4,2 \pm 1,4$ & $4,2 \pm 0,8$ & $5,0 \pm 0,5$ \\
3 & 25 & $0,4 \pm 0,3$ & $0,8 \pm 0,05$ & $0,1 \pm 0,05$ \\
\hline
\end{tabular}

\footnotetext{
* Note : un seul pack de lait ayant été fourni pour ce type de lait, l'analyse a été répétée deux fois. Pour les autres échantillons, les analyses ont été réalisées sur chacun des trois packs de lait.

A single milk sample was provided for this type of milk, the analysis was carried out twice. For the other samples, analysis were performed on the three milk samples.
} 
sement. Elle est déjà inférieure à la concentration attendue après un mois de conservation à $4{ }^{\circ} \mathrm{C}$. De manière générale, la concentration en vitamine $C$ est toujours plus élevée à $4{ }^{\circ} \mathrm{C}$ qu'à $25^{\circ} \mathrm{C}$ et diminue essentiellement jusqu'à deux mois de conservation, la chute étant plus lente ensuite. Après trois mois de conservation il ne reste pratiquement plus de vitamine $\mathrm{C}$ dans les laits conservés à $25^{\circ} \mathrm{C}$.

\section{2. Évolution de la réaction de Maillard en fonction de la température et de la durée de conservation}

\subsubsection{Furosine}

Le tableau III présente les concentrations de furosine mesurées dans chacun des laits au cours des trois mois de stockage. Celleci est deux fois plus élevée dans le LC par rapport à $L V$, mais seulement 1,5 fois plus élevée dans le nouveau lait sans lactose ajouté. La furosine est stable au cours des trois mois de conservation à $4{ }^{\circ} \mathrm{C}$, mais augmente régulièrement $(+50 \%$ en deux mois) lorsque la conservation se fait à $25^{\circ} \mathrm{C}$, sauf pour le LCM pour lequel la furosine n'évo-

Tableau III. Concentration de furosine (mg. $100 \mathrm{~g}^{-1}$ de protéines) dans les laits.

Table III. Furosine concentration $\left(\mathrm{mg} \cdot 100 \mathrm{~g}^{-1}\right.$ of protein) in the milk samples.

\begin{tabular}{lcccc}
$\begin{array}{l}\text { Conservation } \\
\text { (mois) }\end{array}$ & Température & $\begin{array}{l}\text { LV } \\
\left({ }^{\circ} \mathrm{C}\right)\end{array}$ & LC & LCM \\
\hline 1 & 4 & 114 & 204 & 151 \\
1 & 25 & 136 & 232 & 199 \\
2 & 4 & 128 & 232 & 191 \\
2 & 25 & 151 & 244 & 189 \\
3 & 4 & 107 & 218 & 153 \\
3 & 25 & 185 & 309 & 210
\end{tabular}

Note : un seul pack de lait a été analysé sur les trois exemplaires fournis.

A single sample was analysed out of the three samples provided. lue pas significativement. Cependant, nous observons une concentration légèrement plus élevée à $25^{\circ} \mathrm{C}$ qu'à $4{ }^{\circ} \mathrm{C}$ qui laisse supposer que le premier mois de conservation a eu un effet. L'augmentation transitoire significative de la concentration de furosine à deux mois pour le LCM conservé à $4{ }^{\circ} \mathrm{C}$ semble aberrante.

\subsubsection{Les produits avancés de Maillard}

Le tableau $I V$ indique les valeurs FAST. Celles-ci correspondent à la fluorescence des produits avancés de Maillard des protéines solubles dans le tampon acétate à $\mathrm{pH} 4,6$, corrigée de la fluorescence du Trp. La valeur FAST a légèrement tendance à diminuer au cours de la conservation mais est identique quelle que soit la température de conservation. L'augmentation transitoire de la valeur pour le LC conservé deux mois à $4{ }^{\circ} \mathrm{C}$ semble de l'ordre de l'erreur, importante sur cet échantillon. Elle est deux fois plus élevée dans LC que dans LV et est quatre fois plus faible dans LCM par rapport à LC.

\subsection{Composition protéique et teneurs en Trp et Tyr des surnageants acétate des trois types de lait}

La composition protéique des surnageants acétate à pH 4,6 a été analysée sur les trois types de lait conservés trois mois. Le tableau $V$ indique les concentrations en protéines totales et en $\alpha$-lactalbumine et $\beta$-lactoglobuline de ces surnageants. Le chromatogramme d'exclusion obtenu par détection fluorimétrique à $280 / 337 \mathrm{~nm}$ fait apparaître deux pics largement majoritaires à des temps de rétention correspondant aux poids moléculaires de la $\beta$-lactoglobuline (environ $18000 \mathrm{~g} \cdot \mathrm{mol}^{-1}$ ) et de l' $\alpha$-lactalbumine (environ $14000 \mathrm{~g} \cdot \mathrm{mol}^{-1}$ ). En revanche, lorsque la détection se fait en UV (280 nm), d'autres pics de forte amplitude apparaissent correspondant à de plus faibles poids moléculaires (3000 à $\left.12000 \mathrm{~g} \cdot \mathrm{mol}^{-1}\right)$. Cette fraction peptidique ne semble donc pas 
Tableau IV. Valeurs FAST (cps/cps). Fluorescence des produits avancés de Maillard ramenée à la fluorescence du Trp.

Table IV. FAST values (cps/cps). Fluorescence of advanced Maillard products divided by the Trp fluorescence.

\begin{tabular}{lrccl}
\hline $\begin{array}{l}\text { Conservation } \\
\text { (mois) }\end{array}$ & $\begin{array}{c}\text { Température } \\
\left({ }^{\circ} \mathrm{C}\right)\end{array}$ & $\begin{array}{c}\mathrm{LV} \\
\mathrm{N}=3\end{array}$ & $\begin{array}{c}\mathrm{LC} \\
\mathrm{N}=3\end{array}$ & $\begin{array}{c}\text { LCM } \\
\mathrm{N}=2^{*}\end{array}$ \\
\hline 1 & 4 & $128 \pm 2,1$ & $233 \pm 23,7$ & $65 \pm 1,4$ \\
1 & 25 & $131 \pm 3,2$ & $236 \pm 12,1$ & $69 \pm 6,6$ \\
2 & 4 & $112 \pm 9,4$ & $263 \pm 24,5$ & 53 \\
2 & 25 & $119 \pm 11,5$ & $204 \pm 15,9$ & 64 \\
3 & 4 & $100 \pm 9,2$ & $205 \pm 11,0$ & $55 \pm 0,3$ \\
3 & 25 & $96 \pm 2,6$ & $220 \pm 2,5$ & $56 \pm 6,5$ \\
\hline
\end{tabular}

Note : un seul pack de lait ayant été fourni pour ce type de lait, l'analyse a été répétée deux fois. Pour les autres échantillons, les analyses ont été réalisées sur chacun des trois packs de lait.

A single milk sample was provided for this type of milk, the analysis was carried out twice. For the other samples, analysis were performed on the three milk samples.

Tableau V. Composition protéique des surnageants acétate.

Table V. Protein composition of the acetate supernatants.

\begin{tabular}{lccc}
\hline $\begin{array}{l}\text { Analyse } \\
\text { (mois) }\end{array}$ & $\begin{array}{c}\mathrm{LV} \\
n=2\end{array}$ & $\begin{array}{c}\mathrm{LC} \\
n=2\end{array}$ & $\begin{array}{l}\mathrm{LCM} \\
n=2\end{array}$ \\
\hline Protéines totales $\left(\mathrm{g} \cdot \mathrm{L}^{-1}\right)$ & $2,59 \pm 0,43$ & $2,72 \pm 1,7$ & $3,02 \pm 1,27$ \\
$\alpha$-lactabumine $\left(\mathrm{g} \cdot \mathrm{L}^{-1}\right)$ & $0,165 \pm 0,010$ & $0,153 \pm 0,016$ & $0,156 \pm 0,013$ \\
$\beta$-lactoglobuline $\left(\mathrm{g} \cdot \mathrm{L}^{-1}\right)$ & $0,053 \pm 0,07$ & $0,063 \pm 0,007$ & $0,085 \pm 0,015$ \\
\hline
\end{tabular}

contenir de Trp, et doit correspondre à une partie de la fraction protéose-peptone.

Dans les laits de grande consommation soumis à divers traitements thermiques, il existe une relation linéaire entre la fluorescence du Trp (FW) et la concentration en protéines (PS) du surnageant acétate [3,4]. L'équation de la droite de régression est du type FW = a (PS-b). Le facteur constant b correspond à la fraction protéique résistante au traitement thermique et qui ne présente pas de fluorescence Trp. Il semble donc que ce coefficient corresponde à la concentration moyenne de la fraction protéose-peptone dans les laits.
Pour évaluer l'intensité relative du Trp dans les seules protéines du surnageant acétate contenant cet acide aminé (donc essentiellement dans l' $\alpha$-lactalbumine et la $\beta$-lactoglobuline), nous avons choisi de diviser l'intensité de fluorescence du Trp mesurée sur le surnageant acétate (mesure obtenue dans le cadre de la méthode FAST) par le facteur PS-b. Pour le lait UHT LV, cette valeur correspond à la pente de la droite de régression «a ». Cette constante est ramenée à 100 , ce qui permet de s'affranchir des fluctuations de la lampe de fluorescence au cours du temps et de calculer le rendement de fluorescence du Trp dans les laits de 
Tableau VI. Concentrations de Trp et Tyr et fluorescence relative du Trp dans les protéines totales et solubles des laits.

Table VI. Trp and Tyr concentrations and relative Trp fluorescence in total and soluble milk proteins.

\begin{tabular}{lccc}
\hline $\begin{array}{l}\text { Analyse } \\
\text { (mois) }\end{array}$ & $\begin{array}{c}\text { LV } \\
n=2\end{array}$ & $\begin{array}{c}\text { LC } \\
n=2\end{array}$ & $\begin{array}{c}\text { LCM } \\
n=2\end{array}$ \\
\hline Trp total $\left(\mathrm{mg} \cdot \mathrm{g}^{-1}\right)$ & $13,97 \pm 0,33$ & $14,88 \pm 0,82$ & $14,59 \pm 0,54$ \\
Tyr total $\left(\mathrm{mg} \cdot \mathrm{g}^{-1}\right)$ & $43,75 \pm 0,98$ & $47,01 \pm 2,51$ & $46,42 \pm 1,73$ \\
Trp soluble $\left(\mathrm{mg} \cdot \mathrm{g}^{-1}\right.$ Protéines solubles) & $7,8 \pm 0,1$ & $8,4 \pm 1,1$ & $5,3 \pm 0,85$ \\
Tyr soluble $\left(\mathrm{mg} \cdot \mathrm{g}^{-1}\right.$ protéines solubles) & $19,2 \pm 0,6$ & $19,3 \pm 2,21$ & $13,7 \pm 0,8$ \\
Trp soluble $\left(\mathrm{mg} \cdot \mathrm{g}^{-1} \alpha \mathrm{L}+\beta \mathrm{L}\right)$ & $20,2 \pm 0,6$ & $24,4 \pm 2,2$ & $15,8 \pm 1,7$ \\
Tyr soluble $\left(\mathrm{mg} \cdot \mathrm{g}^{-1} \alpha \mathrm{L}+\beta \mathrm{L}\right)$ & $49,5 \pm 1,5$ & $56,4 \pm 2,6$ & $40,8 \pm 4,5$ \\
F (Trp) $/ \mathrm{g}$ PS $(\%)$ & $94,0 \pm 5,7$ & $90,1 \pm 0,5$ & $21,4 \pm 0,8$ \\
\hline
\end{tabular}

Note : les dosages ont été réalisés sur les échantillons de lait à trois mois uniquement. Les valeurs des packs conservés à $4^{\circ} \mathrm{C}$ et à $25^{\circ} \mathrm{C}$ ont été regroupées (non significativement différents).

The analyses were performed only on the milk samples preserved for three months. The values obtained on the samples preserved at $4{ }^{\circ} \mathrm{C}$ and $25^{\circ} \mathrm{C}$ were pooled (not significantly different).

croissance en pourcentage (tableau VI). On $s$ 'aperçoit alors que la fluorescence relative du Trp est diminuée de trois fois dans LC par rapport à LV, alors que dans LCM elle se rapproche de celle de LV.

L'analyse HPLC des résidus Trp et Tyr dans ces surnageants indique en effet une diminution de $30 \%$ du nombre de résidus Trp et Tyr dans LC par rapport à LV, mais non dans LCM. Afin de s'assurer que cette diminution n'est pas due à une baisse de la concentration relative en protéines riches en Trp dans le surnageant de LC, la concentration du Trp a été ramenée à la concentration d' $\alpha$-lactalbumine et $\beta$-lactoglobuline solubles à $\mathrm{pH} 4,6$, excluant ainsi la contribution de la fraction protéose-peptone dépourvue de cet acide-aminé. La concentration relative de Trp est alors plus élevée pour tous les types de lait, conformément aux attentes, mais demeure encore, dans le lait LC, inférieure de $25 \%$ à la concentration de LV, alors que LCM conserve une concentration similaire, voire supérieure, à celle de LV. La concentration relative de tyrosine dans les deux protéines sériques est également diminuée dans LC par rapport aux deux autres types de lait. Lorsque le dosage du Trp et de la Tyr peptidiques est réalisé sur les protéines totales des trois types de lait, la concentration de ces acides aminés est similaire dans les trois types de lait.

\subsection{Comparaison des laits de l'étude avec les autres laits de croissance du commerce}

Pour illustrer la variabilité de la qualité des laits de croissance fournis dans le commerce, deux paramètres ont été utilisés : la mesure FAST, marqueur de la réaction avancée de Maillard et la « fluorescence relative du Trp » dans la fraction soluble des laits à $\mathrm{pH} 4,6$, qui reflète la diminution de fluorescence de cet acide aminé dans les protéines du lactosérum (figure 1). Le lait LV, comme l'ensemble des laits UHT, pour lesquels la fluorescence relative du Trp reste stable, se situe à l'extrémité droite de la figure, La seule variation observée pour les laits de UHT est de type vertical (variation de la valeur FAST uniquement). À l'inverse, dans les laits de croissance, l'augmentation de la valeur FAST s'accompagne d'une 


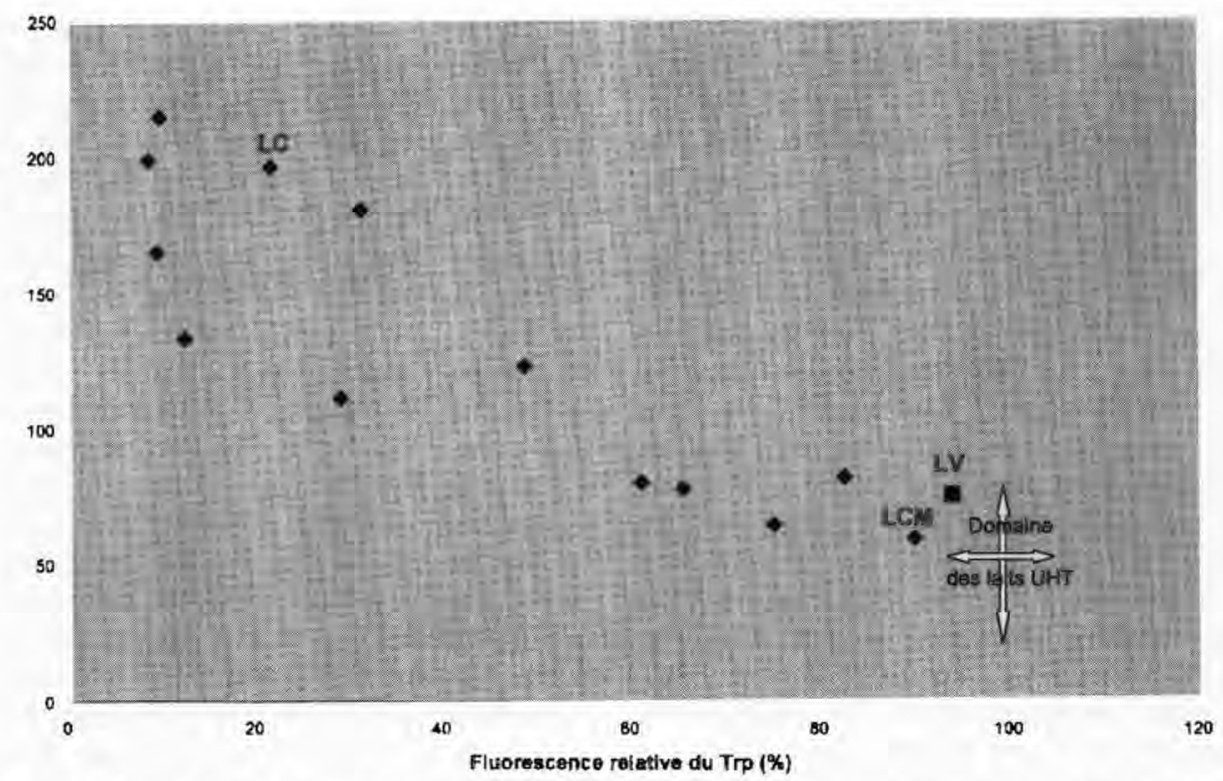

Figure 1. Évaluation de la qualité des laits de croissance du commerce par la méthode globale FAST. La fluorescence du tryptophane et des produits avancés de Maillard ont été dosés comme décrits en méthodes sur les surnageants acétate de divers laits de croissance du commerce et sur les laits de l'étude. En abscisse, la fluorescence relative du tryptophane par g de protéines contenant cet acide aminé est donnée en pourcent. Le domaine des laits UHT est matérialisé par deux flèches qui indiquent les variations de FAST et de fluorescence relative du Trp obtenus à partir de 47 laits UHT du commerce d'après [4].

Figure 1. Evaluation of the quality of commercial growth milk using the global method FAST. The fluorescence of Trp and of advanced Maillard products were measured as described in Methods on the acetate supernatant of various commercial growth milk samples. The LV, LC and LCM samples are indicated. The relative Trp fluorescence is given on the $\mathrm{x}$-axis as a percentage of the mean value obtained in UHT milk samples according to [4]. The distribution of UHT milk samples is indicated by two arrows illustrating the variations in the FAST level and in the relative Trp fluorescence obtained on 47 commercial UHT milk samples [4].

diminution de la valeur de fluorescence relative du Trp. Le lait LCM se situe à l'extrémité droite et basse de la figure. Le lait LC se situe pour sa part dans la partie gauche et haute de la figure.

\section{DISCUSSION}

La stérilisation des aliments, dont le traitement UHT des laits, s'accompagne inévitablement d'une perte de qualité nutritionnelle. Les principales conséquences du traitement thermique sont la dégradation des vitamines les plus fragiles (vitamine C) et le développement de la réaction de Maillard. La formulation particulière des laits de croissance entraîne une amplification de ces réactions de dégradation, notamment en raison de l'enrichissement en lactose, fer et vitamine C [3]. La qualité de ces laits se trouve être en conséquence très inférieure à celle des laits de vache UHT pour adultes [5]. Ceci est difficilement acceptable étant donné le rôle nutritionnel que veulent jouer ces laits et les besoins plus stricts des jeunes enfants en matière de nutrition et de sécurité alimentaire. 
Outre le blocage irréversible de la lysine lié à la réaction de Maillard [10], l'addition du mélange fer-vitamine $C$ entraîne la production d'espèces radicalaires de l'oxygène [2] qui activent la réaction avancée de Maillard et peuvent oxyder les acides aminés sensibles du lait, en particulier le tryptophane et la tyrosine $[13,16,21]$. En effet, nous confirmons des résultats déjà obtenus sur divers laits du commerce, c'est-à-dire une réduction de la disponibilité de la lysine et un niveau de réaction avancée de Maillard deux fois plus important dans le lait de croissance que dans le lait UHT. En effet, le fer favorise la formation de groupements ènediol en position 2-3 sur le glucose de la molécule de lactose, groupements très réactifs qui conduisent à la formation des déoxysones, propagateurs de la réaction de Maillard $[12,16]$. Les laits de croissance et les formules infantiles peuvent également être sujets à des réactions d'oxydation, en raison de l'action catalytique du fer sur un certain nombre de réactions d'oxydation radicalaire, notamment la formation du radical hydroxyle à partir de la vitamine $\mathrm{C}$ et l'oxydation des acides aminés aromatiques. Ces réactions oxydatives peuvent favoriser l'activité mutagène de certains composés de Maillard [14].

Nous retrouvons en effet à la fois une diminution de la fluorescence relative du Trp et une diminution de concentration de Trp et de Tyr dans le surnageant acétate des laits à $\mathrm{pH} \mathrm{4,6.} \mathrm{Mais} \mathrm{on} \mathrm{ne} \mathrm{peut} \mathrm{exclure} \mathrm{le}$ fait qu'une augmentation relative de la fraction protéose-peptone et autres peptides dépourvus de Trp dans le surnageant acétate explique la baisse de concentration de Trp. En effet, cette fraction est résistante au traitement thermique, alors que les protéines riches en $\operatorname{Trp}(\alpha$-lactalbumine et $\beta$-lactoglobuline) précipitent rapidement au cours de leur dénaturation thermique. C'est pourquoi, nous avons d'une part mesuré la concentration des ces deux protéines dans les surnageants à $\mathrm{pH}$ 4,6 et d'autre part, ramené la concentration du Trp à la teneur en ces deux protéines. Nous montrons que la concentration des deux protéines sériques est similaire dans tous les types de lait. Ainsi, la baisse de concentration du Trp mesurée sur le surnageant acétate total de LC persiste lorsque qu'elle est ramenée à la teneur résiduelle des deux protéines sériques majoritaires. Il semble donc bien que le Trp et la Tyr soient partiellement dégradés dans les protéines sériques du lait de croissance par rapport au lait de vache, mais non dans le lait de croissance modifié. La dégradation du Trp peptidique ne dépasse pas 25-30\% et n'apparaît que dans les protéines solubles qui représentent une très faible part des protéines totales. Cellesci sont en effet les plus sensibles en raison de l'exposition des résidus hydrophobes au milieu environnant que favorise le déplissement de la protéine [16]. Ceci explique que nous ne soyons pas en mesure de la retrouver lorsque la totalité des protéines du lait sont analysées.

On peut s'interroger sur les répercussions nutritionnelles de ces réactions qui doivent entraîner une diminution de la biodisponibilité de la lysine et, dans une moindre mesure, du tryptophane. L'excès de protéines présent dans le lait de vache par rapport aux besoins du jeune enfant doit cependant minimiser ces effets. Mais, une baisse de la concentration des protéines dans les laits infantiles est préconisée actuellement [1], ce qui exigera alors de contrôler très précisément la biodisponibilité des acidesaminés modifiés au cours de ces réactions.

Par ailleurs, les nombreux composés nouveaux qui sont produits dans ces formules à la suite de réactions complexes sont mal connus et soulèvent donc des questions de sécurité alimentaire. En particulier, même si le taux de dégradation du Trp est très faible, la mutagénicité importante des produits d'oxydation de cet acide aminé exige que les conséquences toxicologiques de ces modifications soient éclaircies.

Un compromis doit donc être trouvé entre l'intérêt nutritionnel de la formulation et la qualité du produit. La non supplémentation 
des laits de croissance en lactose et en vitamine $C$ permet de réduire très notablement la production de ces nouveaux composés. En effet, si l'on exclut le niveau encore légèrement plus élevé de furosine dans le lait de croissance nouvelle formule par rapport au lait de vache, tous les autres paramètres dosés se retrouvent aux concentrations mesurées dans le lait de vache. S'agissant des produits avancés de Maillard, on observe même une diminution que nous ne pouvons expliquer par la nouvelle formulation. $\mathrm{Ce}$ lait de croissance modifié se trouve donc parmi les meilleurs du marché, tant sur le critère de la réaction de Maillard, appréhendée par la fluorescence relative des produits avancés, que sur celui de l'intégrité de la structure des protéines solubles, mesurée au travers de la fluorescence relative du tryptophane. À ce titre, la mesure FAST constitue une approche analytique globale de la qualité des laits, permettant de rendre compte de ces deux critères importants d'évaluation des dommages subis par les laits de croissance. Les marges de progrès sont considérables, comme en atteste la grande variabilité de ces deux marqueurs dans les laits du commerce.

En conclusion, l'absence d'enrichissement en lactose et en vitamine $C$ d'un lait de croissance limite très sensiblement la dégradation de la qualité des protéines du lait et la génération de substances indésirables.

\section{REMERCIEMENTS}

Nous remercions la société Nactalia (France) pour nous avoir fourni les échantillons de lait de croissance.

\section{RÉFÉRENCES}

[1] Akeson P.M., Axelsson I.E., Raiha N.C.R., Growth and nutrient intake in three- to twelvemonth-old infants fed human milk of formulas with varying protein, J. Pediatr. Gastroenterol. Nutr. 26 (1998) 297-304.

[2] Almaas R., Rootwelt T., Oyasaeter S., Saugstad O.D., Ascorbic acid enhances hydroxyl rad- ical formation in iron-fortified infant cereals and infant formulas, Eur. J. Pediatr. 156 (1997) 488-492.

[3] Birlouez-Aragon I., Moreaux V., Nicolas M., Ducauze CJ., Effect of iron and lactose supplementation of milk on the Maillard reaction and tryptophan content, Food Addit, Contam. 14 (1997) 381-388.

[4] Birlouez-Aragon I., Nicolas M., Metais A., Marchond M., Grenier J., Calvo D., A rapid fluorimetric method to estimate the heat treatment of liquid milk, Int. Dairy J. 8 (1998) 771-777.

15] Birlouez-Aragon I., Effect of iron fortification on protein nutritional quality of infant and growth formulas, in: Pandalai G. (éd.), Recent Advances in Agricultural and Food Chemistry, Research Signpost, India, 1999, pp. 139-148.

[6] Evangelisti F., Calcagno C., Zunin P., Relationship between blocked lysine and carbohydrate composition of infant formulas, J. Food Sci. 59 (1994) 335-337.

[7] Fazzolari-Nesci A., Domianello D., Sotera V., Räihä NCR., Tryptophan fortification of adapted formula increases plasma trytophan concentrations to levels not different from those found in breast-fed infants, J. Pediat. Gastroenterol. Nutr. 14 (1992) 456-459.

[8] Garcia R., Alegria A., Barbera R., Farre R., Lagarda MJ., Dializability of iron, zinc, and copper of different types of infant formulas marketed in Spain, Biol. Tr. Elem. Res. 65 (1998) 7-17.

[9] Gill D., Vincent S., Segal D.S., Follow-on formula in the prevention of iron deficiency: a multicenter study, Acta Paediatr. 86 (1997) 683-689.

[10] Henle T., Walter H, Klostermeyer H., Evaluation of the extent of the early Maillard reaction in milk products by direct measurement of the Amadori product lactulosyl-lysine, Lebensm. Unters. Forsch. 193 (1991) 19-22.

[11] Hercberg S., Preziosi P., Galan P., Deheeger M., Papoz L., Dupin H., Apports nutritionnels d'un échantillon représentatif de la population du Val-de-Marne : III Les apports en minéraux et vitamines, Rev. Epidemiol. Santé Publ. 39 (1991) 245-261.

[12] Kato Y., Watanabe K., Sato Y., Effect of some metals on the Maillard reaction of ovalbumin. J. Agric. Food Chem. 29 (1981) 540-543.

[13] Kawakishi S., Okawa Y., Uchida K., Oxidative damage of protein induced by the Amadori compound-copper ion system, J. Agric. Food Chem. 38 (1990) 13-17.

[14] Kim S.B., Kim I.S., Yeum D.M., Park Y.H., Mutagenicity of Maillard reaction products from D-glucose-amino acid mixtures and possible roles of active oxygens in the mutagenicity, Mutat. Res. 254 (1991) 65-69. 
[15] Landry J., Delhaye S., Simplified procedure for the determination of tryptophan of foods and feedstuffs from barytic hydrolisis, J. Agric. Food Chem. 40 (1992) 776-779.

[16] Moreaux V., Birlouez-Aragon I., Degradation of tryptophan in heated $\beta$-lactoglobulin-lactose mixtures is associated with intense Maillard reaction, J. Agric. Food Chem. 45 (1997) 1905-1910.

[17] Resmini P., Pellegrini I., Batelli G., Accurate quantification of furosine in milk and dairy products by a direct HPLC method, Ital. J. Food Sci. 3 (1990) 173-183.

[18] Rudloff S., Lönnerdal B., Solubility and digestibility of milk proteins in infant formulas exposed to different heat treatment, J. Pediat. Gastroenterol. Nutr. 15 (1992) 25-33.

[19] Sarwar G., Peace R.W., Botting H.G., Differences in protein digestibility and quality of liq- uid concentrate and powder forms of milk-based infant formulas fed to rats, Amer. J. Clin. Nutr. 49 (1989) 806-813.

[20] Tessier F., Birlouez-Aragon I., Tjani C., Guilland J.C., Validation of a micromethod for determining oxidized and reduced vitamin $C$ plasma by HPLC-fluorescence, Int. J. Vit. Nutr. Res. 66 (1996) 166-170.

[21] Uchida K., Kawakishi S., Selective oxidation of tryptophan and histidine residues in proteins through the copper-catalyzed autooxidation of L-Ascorbic acid, Agric. Biol. Chem. 52 (1988) 1529-1535.,

[22] Uchida K., Enomoto N., Itakura K., Kawakishi S., The Hydroxyl radical generated by an iron(II)/EDTA/ascorbate system preferentially attacks tryptophan residues of protein, Agric. Biol. Chem. 53 (1989) 3285-3292. 